\title{
Prevalence of Heart Diseases in Pregnant Women Referred to the Heart Diseases Clinic for Obstetrics
}

\author{
Rozita Hossinzadeh ${ }^{1}$, Razieh Parizad ${ }^{1,2}$, Mehrnoush Toufan Tabrizi $^{1}$, Zeinab Ghasemian Khojasteh ${ }^{*}$, Nava Ainehchi ${ }^{3}$
}

\begin{abstract}
Objectives: Heart diseases are among the most common diseases, subjecting the maternal and fetal life to risks, causing complications for both mothers and babies. Despite medical and surgical progress made in the heart diseases, coronary artery diseases are still the second cause of the mortality and significant disability, as well as the low efficiency in the women over 40 years of age; also, they are the leading cause of mortality in the women over 65 years old. This study was conducted to investigate the prevalence of heart diseases in the pregnant women.

Materials and Methods: This study was a descriptive cross-sectional conducted on the pregnant women with heart problems using the census method from September 2015 until September 2017. After data collection, they were analyzed using SPSS version 17. Results: The findings of the present study showed that dyspnea (28.1\%), cardiac palpation (32.7\%) and hypertension (11.7\%) were the most common causes of pregnant women's referral to obstetrics clinics. Also, most cardiac problems were associated with valvular problems (24.3\%) and heart failure (18\%). The average age of the patients was $28 \pm 9$ years.

Conclusion: Heart diseases are very dangerous during pregnancy, but its progression and complications in the mother and the fetus can be prevented by treatment and continuous, as well as, a complete care during pregnancy and before delivery.

Keywords: Pregnancy, Heart disease, Women's health
\end{abstract}

\section{Introduction}

Pregnancy and childbirth have been recognized as a sweet, but a dangerous stage in the culture of the public and their minds (1). The mother, the main core of the family, is the element of health and success for other members (2). Pregnancy causes changes in the different systems of her body, especially in the cardiovascular system, which they are necessary for a successful pregnancy and the birth of a healthy baby. Heart diseases and pregnancy increase the number of maternal mortalities (3). Maternal mortality rate is an index for national development and the reason for choosing this index is one of the most prominent indicators of development since it is affected by various social and economic factors (4).

The frequency of heart disease in pregnancy has fallen from $3 \%$ to $1 \%$; this is important in terms of their bad interaction, such that serious cardiac diseases increase the incidence of abortion, intrauterine death, preterm labor, and intrauterine growth retardation, and in severe cases, the fetal death reaches to $20 \%-40 \%$, while the maternal mortality reaches to about $5 \%$ in the intermediate cases and $25 \%-50 \%$ in the most severe and dangerous cases (5).

Today, the most pregnant women with cardiac diseases have mild recovered congenital cardiac disease. Although rheumatic valvular diseases have been seen in pregnant women in developing countries in the past, other morbidities, such as cardiomyopathies, mitral and aortic valve prolapses, pulmonary arterial hypertension, and coronary artery diseases are seen less frequently (6). Approximately 1 million people in the Western countries have congenital heart disease, of which more than half are women in their fertile years (7.8). Coronary artery disease is the second cause of significant mortality and disability, as well as low productivity in the women over 40 , and also the first cause of mortality in the women over 65 years (6).

Heart diseases and pregnancy increase the maternal mortality, which is considered as one of the leading development indicators in every country. In addition, maternal mortality imposes high costs for the family and the society (9). Since most cardiac and hemodynamic indices, such as increased heart rate and cardiac output, as well as reduced peripheral vascular resistance are significantly altered in the pregnancy and all these factors and changes can lead to maternal heart disease or its exacerbation, endangering the life of the mother and the fetus (10), maternal health always has the highest priority in the healthcare system. On the other hand, there are many serious diseases and risk factors that can be identified with the lowest cost and conveniently, using pre-pregnancy care in order to prevent their occurrence or impact on pregnancy. Heart diseases are among these risk factors which their occurrence and progress can be 
prevented by early detection and diagnosis $(11,12)$.

Given to the lack of study in this area and the importance of the issue of women $>$ health, especially during pregnancy, we aimed to evaluate the prevalence of heart diseases in those pregnant women who referred to the heart diseases clinic for obstetrics.

\section{Materials and Methods}

The present study was a descriptive cross-sectional conducted on 90 pregnant women with heart disease in Clinic for Obstetrics in Tabriz from September 2015 until September 2017. Sampling has been carried out using the accessible sampling method. A researcher-made questionnaire was used for data collection, which consists of 2 parts: the first part includes demographic information and the second part is related to heart disease. The data were analyzed using SPSS statistical software version 17.

\section{Results}

During the study, 90 pregnant women were surveyed, of which $62(55.8 \%)$ lived in cities and 28 (25.2\%) lived in rural areas. The average age of the patients was $28 \pm 9$. Of all studied subjects, 28 (25.2\%) were illiterate, 25 (22.5\%) had an education under high school diploma, 19 (17.1\%) had a high school diploma, 10 (9\%) had bachelor degree, and 8 (2.7\%) had master degree (Table 1). Also, of all the subjects, 67 (63.3\%) were housewives and 23 (20.7\%) were employees. The most frequent causes of pregnant women's hospitalization were dyspnea (28.1\%) and cardiac palpation (32.7\%) (Table 2). The valvular heart complications are considered as the most frequent problems in the medical history of pregnant women (Table 3). The most common heart disease among patients was mitral valvel regurgitation (21.6\%) (Table 4). Most patients did not have a history of admission (Table 5). Among the risk factors, the highest frequency was related to high blood pressure $(11.7 \%)$ and the lowest rate of smoking (Table 6). The most commonly used drugs among patients were beta blocker $(25.8 \%)$ and the most treatment was for non-cardiac patients (Tables 7 and 8). Seven oercent of patients died during labor (Table 9). The highest rate of hospitalization in the pregnant women

Table 1. Demographic Data of the Studied Population

\begin{tabular}{lll}
\hline Variables & Nol (\%) \\
\hline \multirow{3}{*}{ Education } & Under high school diploma & $25(22.5)$ \\
& High school diploma & $19(17.1)$ \\
& Bachelor degree & $9(9.0)$ \\
& Master degree & $8(7.2)$ \\
\hline \multirow{2}{*}{ Living Place } & Cities & $62(55.8)$ \\
& Rural areas & $28(25.2)$ \\
\hline \multirow{2}{*}{ Occupation } & Housewife & $67(60.3)$ \\
& Employee & $23(20.7)$
\end{tabular}

Table 2. Frequency Distribution of the Leading Cause of Referral by the Pregnant Women to Obstetrics Clinics

\begin{tabular}{lc}
\hline Variables & No. (\%) \\
\hline Dizziness & $2(2.2)$ \\
Coughing and edema & $3(3.3)$ \\
Dyspnea & $29(21.1)$ \\
Sweating & $2(2.2)$ \\
Chronic high blood pressure in the field of disease & $8(9.2)$ \\
Diabetes & $5(5.5)$ \\
Cardiac Palpation & $33(32.7)$ \\
Headache & $2(2.2)$ \\
Chest pain during rest & $4(4.4)$ \\
Dyspnea during activity & $2(2.2)$ \\
Preeclampsia & $1(1.6)$ \\
\hline
\end{tabular}

Table 3. Frequency Distribution of Medical History of Pregnant Women

\begin{tabular}{lc}
\hline Variables & No. (\%) \\
\hline Valvular problems & $27(24.3)$ \\
History of high blood pressure & $13(11.7)$ \\
Diabetes & $5(4.5)$ \\
History of heart failure & $20(18)$ \\
Positive family history & $10(9)$ \\
Stable angina & $8(7.1)$ \\
Myocardial Infarction & $7(6.3)$ \\
\hline
\end{tabular}

Table 4. Distribution of Heart Disease in Pregnant Women

\begin{tabular}{lc}
\hline Variables & No. (\%) \\
\hline Mitral regurgitation & $24(21.6)$ \\
History of mitral surgery & $12(10.8)$ \\
Aortic aneurysm & $9(8.1)$ \\
Angina pectoris & $7(6.3)$ \\
Aortic insufficiency & $10(9)$ \\
Congenital anomaly & $11(9.9)$ \\
Cardiomyopathy & $4(3.6)$ \\
Fallot tetralogy & $4(3.6)$ \\
Sinus Tachycardia & $5(4.5)$ \\
Myocardial infarction & $4(3.6)$ \\
\hline
\end{tabular}

Table 5. Frequency Distribution of Hospitalization in Pregnant Women

\begin{tabular}{lc}
\hline Admission Times & No.(\%) \\
\hline Without admission & $38(38.7)$ \\
Once & $25(33.8)$ \\
Twice & $17(12.2)$ \\
Three times & $6(9.8)$ \\
Four times and more & $4(5.9)$ \\
\hline
\end{tabular}


Table 6. Frequency Distribution of Risk Factors in Pregnant Women

\begin{tabular}{lcc}
\hline Drugs & $\begin{array}{c}\text { Yes } \\
\text { No. (\%) }\end{array}$ & $\begin{array}{c}\text { No } \\
\text { No. (\%) }\end{array}$ \\
\hline Cigarette & $2(3.6)$ & $45(88.2)$ \\
Hyperlipidemia & $4(5.8)$ & $68(94.1)$ \\
Diabetes & $5(4.5)$ & $63(53.1)$ \\
Hypertension & $13(11.7)$ & $51(66.1)$ \\
Positive family history & $10(9)$ & $49(78.1)$ \\
History of kidney failure & $3(2.6)$ & $71(97.1)$ \\
History of using Anti- hypertension & $11(14.1)$ & $63(73.9)$ \\
medications & $2(4.6)$ & $71(68.4)$ \\
\hline History of using diabetes medications & & \\
\hline
\end{tabular}

Table 7. Distribution of Drug Use in Pregnant Women

\begin{tabular}{lcc}
\hline Drugs & $\begin{array}{c}\text { Yes } \\
\text { No. (\%) }\end{array}$ & $\begin{array}{c}\text { No } \\
\text { No. (\%) }\end{array}$ \\
\hline Aspirin & $16(19.6)$ & $45(60.2)$ \\
Beta-blocker & $21(25.8)$ & $56(78.3)$ \\
Diuretic & $5(4.5)$ & $40(43.6)$ \\
Anti arrhythmia & $13(11.7)$ & $61(86.1)$ \\
Warfarin & $12(9)$. & $69(88.7)$ \\
Enoxaparin & $3(2.6)$ & $71(97.1)$ \\
Heparin & $11(14.1)$ & $52(73.9)$ \\
\hline
\end{tabular}

Table 8. Frequency Distribution of Treatment History in Pregnant Women

\begin{tabular}{lc}
\hline History of Treatment & No. (\%) \\
\hline Unstable angina & $13(15.1)$ \\
Myocardial infarction & $7(8.2)$ \\
Angioplasty & $9(10.2$ \\
Other causes & $16(18.3$ \\
No history of treatment & $45(85.9)$ \\
\hline
\end{tabular}

Table 9. Frequency Distribution at the End of Pregnancy

\begin{tabular}{lc}
\hline Result of the Disease & No. (\%) \\
\hline Alive & $81(90)$ \\
Death & \\
Eisenmenger's syndrome & $3(3.3)$ \\
Cardiomyopathy and severe heart failure & $3(2.96)$ \\
Severe aortic aneurysm & $2(2.2)$ \\
Pulmonary edema & $1(1.9)$ \\
\hline
\end{tabular}

was dyspnea (28.1\%) and cardiac palpation (32.7\%), respectively and the lowest one was preeclampsia (1.1\%) with cardiac disease (Table 2).

\section{Discussion}

During the period of the study, out of 90 pregnant women under study, 20 had heart failure (18 cases per thousand).
The mean age of the women in the study was $28 \pm 9.5$, which is acceptable with respect to the increased age of marriage, on the one hand and the high number of pregnancies and abortions, on the other hand. The most common cause of hospitalization in the women was dyspnea, cardiac palpitations, and high blood pressure, which the most common cause in the study by Dadgar and Porjavad was dyspnea during exercise (13). Among the mothers with medical histories, $24.3 \%$ reported cardiac valvular problems, $18 \%$ a history of heart failure, and $11.7 \%$ a history of high blood pressure. In the study by Dadgar and Porjavad, the highest frequency of referral to obstetrics clinics by the pregnant women was $13.81 \%$ (13).

In this study, $96 \%$ of women were treated with angiography and medications and only $4 \%$ were under the care, due to risk symptoms. In the study by Shahgheibi and Naghshbandi, the prevalence of heart disease in pregnant women was $1.6 \%$ (14). In this study, mitral regurgitation had the highest prevalence of heart disease. Shahgheibi and Naghshbandi reported that the most common type of heart disease in mothers was mitral stenosis (MS) (22\%) (14). Dadgar and Porjavad highlighted that the rheumatic heart disease was the most common cause of heart disease (96.8\%) (13). Balci et al reported that cardiovascular events in mothers occurred in $10.2 \%$ of the pregnancies (15).

In a study conducted in England in 2002, mitral valve prolapse (MVP) was identified as the most common heart disease, which its common symptom is heart palpitations during pregnancy, and the patients have been treated with Inderal (16). Rheumatic valvular stenosis is still considered as the most common heart disease in the pregnant women in many countries, which is manifested as mitral stenosis in $75 \%$ of the cases (17). Therefore, heart rheumatism has been identified as one of the most common causes of valvular heart disease. Wang et al found that the prevalence of heart rheumatism is equal to $8 / 1000$ (18). Rheumatic heart disease is the most common cause of the existing valvular diseases in females in our country. MVP is often well-tolerated with treatment (19). Congenital heart disease is the most common cause of heart damages in the pregnant women. In the absence of heart disease, the structural basis of cardiac arrhythmias is rare during pregnancy, usually not requiring any medication $(20,21)$. Diagnosing and treatment of heart disease in a timely manner is highly critical during pregnancy. Valvular stenoses are not much well-tolerated than [heart] failures in the pregnancy (22).

\section{Conclusion}

Precise understanding and evaluation of mothers with heart disease are of particular importance. Due to functional and morphological variations of heart disease, it is difficult to predict its complications. Therefore, any complication in pregnant women during pregnancy should be taken into account seriously. Physicians and 
nurses are well aware that females with heart diseases will face high-risk pregnancies, but accurate determination of the quantity and quality of these risks is associated with difficulties for mothers and babies, and this indicates the requirement for a close control and monitoring of pregnancy in mothers with heart disease

\section{Ethical Issues}

The local ethics committee approved the study.

\section{Conflict of Interests}

Authors declare that they have no conflict of interests.

\section{Financial Support}

None.

\section{References}

1. Cunningham FG, Wenstrom K, Hauth J, Leveno K, Gilstrap L. Williams Obstetrics. 21st ed. New York: McGraw Hill; 2001: 1181-1203.

2. Scott R, Disaia G, Harmond B. Donforth 's Obstertics and Gynecology. 8th ed. New York: Williams and Wilkins; 1999: 336-342.

3. Zipes DP, Braunwald E. Pregnancy \& Heart Disease. In: Braunwald's Heart Disease: A Textbook of Cardiovascular Medicine. Saunders Elsevier; 2008.

4. Faiz SA, Al-Meshari AA, Sporrong BG. Pregnancy and valvular heart disease. Saudi Med J. 2003;24(10):1098-1101.

5. Siu SC, Sermer M, Colman JM, et al. Prospective multicenter study of pregnancy outcomes in women with heart disease. Circulation. 2001;104(5):515-521.

6. Stout K. Pregnancy in women with congenital heart disease: the importance of evaluation and counselling. Heart. 2005;91(6):713-714. doi:10.1136/hrt.2004.047886.

7. Fernandes SM, Arendt KW, Landzberg MJ, Economy KE, Khairy P. Pregnant women with congenital heart disease: cardiac, anesthetic and obstetrical implications. Expert Rev Cardiovasc Ther. 2010;8(3):439-448. doi:10.1586/ erc.09.179.

8. Bereck JS. Novak's Gynecology. 13th ed. Los Angeles: Wolters Klumer; 2002.

9. Franklin WJ, Gandhi M. Congenital heart disease in pregnancy. Cardiol Clin. 2012;30(3):383-394. doi:10.1016/j. ccl.2012.04.003

10. Wald RM, Sermer M, Colman JM. Pregnancy and contraception in young women with congenital heart disease: General considerations. Paediatr Child Health. 2011;16(4):e25-29.
11. Siu SC, Colman JM, Sorensen S, et al. Adverse neonatal and cardiac outcomes are more common in pregnant women with cardiac disease. Circulation. 2002;105(18):2179-2184.

12. Harris IS. Management of pregnancy in patients with congenital heart disease. Prog Cardiovasc Dis. 2011;53(4):305-311. doi:10.1016/j.pcad.2010.08.001

13. Dadgar AA, Pourjavad M. Study 7 years in Pregnant Women Whit heart disease. women midwifery and barrenness Iranian Journal .Fall and Winter2004;7(2):84-90.

14. Shahgheibi SH, Naghshbandi M. The prevalence of cardiovascular Disease and Its Effect on pregnancy outcome in pregnant Women. scientific searching Journal in Kordestan. 2007;8(29):45-50.

15. Balci A, Sollie-Szarynska KM, van der Bijl AG, et al. Prospective validation and assessment of cardiovascular and offspring risk models for pregnant women with congenital heart disease. Heart. 2014;100(17):1373-1381. doi:10.1136/heartjnl-2014-305597

16. Khairy P, Ouyang DW, Fernandes SM, Lee-Parritz A, Economy KE, Landzberg MJ. Pregnancy outcomes in women with congenital heart disease. Circulation. 2006;113(4):517524. doi:10.1161/circulationaha.105.589655

17. Yaghoubi A, Pezeshkian M, Imani S, Alizadeye Asl A. Analysis of Maternal-Fetal outcomes of Valvular Heart Surgeries in Pregnant Women. Zahedan Journal of Research in Medical Sciences. 2009;12(1):40-43.

18. Wang X, Chen C, Wang L, Chen D, Guang W, French J. Conception, early pregnancy loss, and time to clinical pregnancy: a population-based prospective study. Fertil Steril. 2003;79(3):577-584.

19. Tateno S, Niwa K, Nakazawa M, Akagi T, Shinohara T, Yasuda T. Arrhythmia and conduction disturbances in patients with congenital heart disease during pregnancy: multicenter study. Circ J. 2003;67(12):992-997.

20. Moeinipour AA, Sepehri Shamloo A, Lotfalizadeh M, Esfahanizadeh J, Mottahedi B, Hoseinikhah H. Evaluation of last guidelines and studies about the best treatment with anticoagulant during pregnancy in woman with mechanical heart valves. Iran J Obstet Gynecol Infertil. 2014;18(139):15-19.

21. Niwa K, Tateno S, Akagi T, et al. Arrhythmia and reduced heart rate variability during pregnancy in women with congenital heart disease and previous reparative surgery. Int J Cardiol. 2007;122(2):143-148. doi:10.1016/j. ijcard.2006.11.045

22. Drenthen W, Pieper PG, Roos-Hesselink JW, et al. Outcome of pregnancy in women with congenital heart disease: a literature review. J Am Coll Cardiol. 2007;49(24):23032311. doi:10.1016/j.jacc.2007.03.027

Copyright (C) 2018 The Author (s); This is an open-access article distributed under the terms of the Creative Commons Attribution License (http://creativecommons.org/licenses/by/4.0), which permits unrestricted use, distribution, and reproduction in any medium, provided the original work is properly cited. 